\title{
A problem of von Neumann and Maharam about algebras supporting continuous submeasures
}

\author{
by \\ Stevo Todorcevic (Paris, Toronto and Bellaterra)
}

\begin{abstract}
We show that a $\sigma$-algebra $\mathbb{B}$ carries a strictly positive continuous submeasure if and only if $\mathbb{B}$ is weakly distributive and it satisfies the $\sigma$-finite chain condition of Horn and Tarski.
\end{abstract}

1. Introduction. Recall that a measure algebra is a complete boolean algebra $\mathbb{B}$ supporting a strictly positive countably additive measure. The problem of characterizing measure algebras in the class of complete boolean algebras was first proposed by von Neumann in the 1930's (see [14]). The first necessary condition isolated in [14] is the countable chain condition asserting that every cellular family of members of $\mathbb{B}$ must be countable. Recall that a cellular family is any family of pairwise disjoint members of $\mathbb{B}$. The second necessary condition given in [14] is the weak distributivity of $\mathbb{B}$ asserting that for every double sequence $a_{n k}$ (indexed by non-negative integers) of elements of $\mathbb{B}$,

$$
\bigwedge_{n} \bigvee_{k} a_{n k}=\bigvee_{F} \bigwedge_{n} a_{n F(n)}
$$

where $F=(F(n))$ ranges over all sequences of finite sets of non-negative integers and where $a_{n F(n)}$ is defined to be the supremum of $a_{n k}$ for $k \in F(n)$. So this is a natural weakening of the usual distributive law, where the $F(n)$ 's are assumed to be all singletons. The first major advance on von Neumann's problem is given by Maharam [11] who correctly identified it as a problem which is in part a metrization problem for the corresponding sequential topology of $\mathbb{B}$ (see Section 2 for the exact definition of this topology). More precisely, Maharam [11] shows that the sequential topology of $\mathbb{B}$ is metrizable if and only if $\mathbb{B}$ supports a strictly positive continuous submeasure. Recall

2000 Mathematics Subject Classification: Primary 28A60.

Research for this article was partially supported by CNRS, NSERC, and Ministerio de Educatión, Cultura y Deporte. 
that a strictly positive continuous submeasure on a $\sigma$-algebra $\mathbb{B}$ is a function $\nu: \mathbb{B} \rightarrow[0, \infty)$ such that:

(a) $\nu(a)=0$ if and only if $a=\mathbf{0}$,

(b) $a \leq b$ implies $\nu(a) \leq \nu(b)$,

(c) $\nu(a \vee b) \leq \nu(a)+\nu(b)$,

(d) $\bigwedge_{n} a_{n}=\mathbf{0}$ implies $\nu\left(a_{n}\right) \rightarrow 0$ for every decreasing sequence $a_{n}$.

If $(d)$ is weakened to

$\left(\mathrm{d}^{-}\right)$if $a_{n}$ is an increasing sequence then $\nu\left(a_{n}\right) \rightarrow \nu\left(\bigvee_{n} a_{n}\right)$,

then one says that $\mathbb{B}$ supports a strictly positive submeasure. A submeasure $\nu$ on $\mathbb{B}$ is non-trivial if for every $a$ in $\mathbb{B}^{+}=\mathbb{B} \backslash\{\mathbf{0}\}$ there is $b \leq a$ such that $0<\nu(b)<\nu(a)$. The work of Maharam [11] gives a natural decomposition of von Neumann's problem into the following two parts:

(I) Does every weakly distributive complete boolean algebra $\mathbb{B}$ satisfying the countable chain condition support a strictly positive continuous submeasure?

(II) Given that $\mathbb{B}$ supports a strictly positive continuous submeasure, does it also support a strictly positive countably additive measure?

Part (II) is the original form of the well-known and well-studied Control Measure Problem which has a strong degree of absoluteness and many reformulations in different areas of Functional Analysis (see [6], [9]). Already at the very start of the analysis of von Neumann's problem it was realized that this formulation of its Part (I) lacks in absoluteness. To explain this, recall that a Suslin algebra is a non-atomic $\sigma$-distributive boolean algebra satisfying the countable chain condition. Its existence is equivalent to the negation of the Suslin hypothesis and is therefore independent of the standard axioms of set theory. In [11], Maharam shows that no Suslin algebra can support a non-trivial submeasure, a result that clearly indicates that the countable chain condition along with weak distributivity may not be sufficient even for the existence of a non-trivial submeasure, at least if one is not willing to go beyond the standard axioms of set theory. Remarkably, quite recently it has been shown by Balcar, Jech and Pazák [3] that the positive answer to (I) is consistent with the standard axioms of set theory. However, one still would like to see if supplementing von Neumann's list with some other necessary conditions one arrives at a positive answer to these questions without going beyond the standard axioms of set theory. It turns out that such a condition can indeed be found, but in a parallel series of investigations that begun with the paper of Horn and Tarski [8] on the following problem of Tarski (see [19], [20]):

(III) Does every boolean algebra satisfying the countable chain condition supports a strictly positive finitely additive measure? 
While working on this problem Horn and Tarski [8] have realized that the countable chain condition has to be strengthened even if one wants to have a finitely additive measure on $\mathbb{B}$. For example, it is readily seen that if $\mu$ is a strictly positive finitely additive measure on $\mathbb{B}$ then the sets $\mathbb{B}_{n}=\{a \in \mathbb{B}$ : $\mu(a)>1 /(n+1)\}$ witness that $\mathbb{B}$ satisfies the following chain condition (the " $\sigma$-bounded chain condition") that is considerably stronger than the countable chain condition:

$(\sigma \mathrm{bcc}) \quad$ There is a decomposition $\mathbb{B}^{+}=\bigcup_{n=0}^{\infty} \mathbb{B}_{n}$ such that for every $n$ the piece $\mathbb{B}_{n}$ of the decomposition contains no cellular subfamily of size $n+2$.

On the other hand, note that if $\nu: \mathbb{B} \rightarrow[0, \infty)$ is a strictly positive continuous submeasure then $\nu$ is exhaustive in the sense that for every $\varepsilon>0$ the set $\{a \in \mathbb{B}: \nu(a)>\varepsilon\}$ contains no infinite cellular family. It follows that every $\sigma$-algebra supporting a strictly positive continuous submeasure satisfies the following chain condition (the " $\sigma$-finite chain condition") which is also considered by Horn and Tarski [8] and which is still considerably stronger than the countable chain condition:

$(\sigma$ fcc $) \quad$ There is a decomposition $\mathbb{B}^{+}=\bigcup_{n=0}^{\infty} \mathbb{B}_{n}$ such that no piece $\mathbb{B}_{n}$ contains an infinite cellular subfamily.

Looking at the difference between $\sigma$ bcc and $\sigma$ fcc one may wonder if there is a finite upper bound on the sizes of cellular subfamilies of $\{a \in \mathbb{B}: \nu(a)>\varepsilon\}$ whenever $\mu$ is a strictly positive continuous submeasure on $\mathbb{B}$ and where $\varepsilon>0$. The positive answer to this question is yet another equivalent formulation of the Control Measure Problem (II) discussed above. Regardless of the state of the Control Measure Problem, one can still ask the following question originally appearing in [8]:

(IV) Is it true that every boolean algebra satisfying the $\sigma$-finite chain condition satisfies in fact the stronger $\sigma$-bounded chain condition?

In [8], Horn and Tarski also ask the following problem:

(V) Is any of the two chain conditions $\sigma$-fcc or $\sigma$-bcc sufficient for the existence of a finitely additive strictly positive measure?

This was answered in the negative by Gaifman [7] using a result of Kelley [10] that characterizes boolean algebras supporting a strictly finitely additive measure. However, neither Horn and Tarski [8], Kelley [10], nor Gaifman [7] discuss the possibility that adding the requirement of weak distributivity to one of these two chain conditions might lead us to the existence of a strictly positive finitely (or countably) additive measure on a given boolean algebra. This version of the Horn and Tarski problem (V) appears explicitly only in Oxtoby's review [15] of Gaifman's paper. More precisely, Oxtoby [15] asks 
the following question:

(VI) Does every weakly distributive boolean algebra satisfying the $\sigma$ bounded chain condition support a strictly positive finitely additive measure?

The following result shows that this might be a right form of the Horn and Tarski problem $(\mathrm{V})$.

THEOREM 1. The following are equivalent for every complete boolean algebra $\mathbb{B}$ :

(1) $\mathbb{B}$ carries a strictly positive continuous submeasure.

(2) (a) $\mathbb{B}$ is weakly distributive and

(b) $\mathbb{B}$ satisfies the $\sigma$-finite chain condition.

It follows, in particular, that von Neumann's problem in the class of $\sigma$-algebras satisfying the $\sigma$-finite chain condition is equivalent to the Control Measure Problem. Moreover, it follows that Oxtoby's question (VI) is in fact yet another reformulation of the Control Measure Problem. It also follows that the positive solution to the Control Measure Problem would also yield a positive answer to the Horn Tarski problem (IV) provided one works in the class of weakly distributive algebras. Recall that the Control Measure Problem is really a problem about countably generated $\sigma$-algebras, i.e., $\sigma$-algebras $\mathbb{B}$ for which there is a countable subset $\mathbb{B}_{0}$ with the property that $\mathbb{B}$ contains no proper $\sigma$-subalgebra containing $\mathbb{B}_{0}$. So it is natural to ask which of the countably generated $\sigma$-algebras support a strictly positive continuous submeasure. This amounts to characterizing the countably generated $\sigma$-algebras that are representable as quotients of the $\sigma$-algebra of Borel subsets of $\mathbb{R}$ over $\sigma$-ideals on $\mathbb{R}$ that have the form

$$
\mathcal{N}_{\nu}=\{X \subseteq \mathbb{R}: \nu(X)=0\}
$$

for some continuous Borel submeasure, or better to say, continuous outer measure $\nu$. In order to analyze this one needs a version of the SikorskiLoomis theorem for weakly distributive and countably generated algebras. This will be done in Section 2 of this note where the corresponding version of Theorem 1 is stated.

2. An ideal of converging sequences. The purpose of this section is to survey the necessary background material needed for the proof of Theorem 1 as well as to state and prove a version of this result for the class of countably generated algebras. Recall the definition of strong convergence of sequences $a_{n}$ of members of some $\sigma$-algebra $\mathbb{B}$ as introduced in [11]:

$$
a_{n} \rightarrow a \quad \text { if and only if } \lim \sup \left(a_{n} \triangle a\right)=\mathbf{0},
$$


where as customary for a sequence $b_{n}$ of members of $\mathbb{B}$ one defines $\lim \sup b_{n}$ to be equal to $\bigwedge_{k} \bigvee_{n>k} b_{n}$. As shown in [11], if $\mathbb{B}$ is weakly distributive this notion of convergence satisfies the axioms of the abstract theory of convergence of Fréchet [5] and, in particular, the following important condition:

If $a_{n} \rightarrow a$ and if $a_{n k}$ is a double sequence such that $a_{n k} \rightarrow a_{n}$ for each $n$ then there is an increasing sequence $k_{n}$ of non-negative integers such that $a_{n k_{n}} \rightarrow a$.

In fact, in the context of $\sigma$-algebras $\mathbb{B}$ satisfying the countable chain condition this principle is equivalent to the weak distributivity of $\mathbb{B}$ as well as to the following diagonal sequence property considered explicitly in many of the early papers on this subject (see [11] and [23]):

(DS) Given a double sequence $a_{n k}$ of members of $\mathbb{B}$ such that for each $n$ the sequence $a_{n k}$ decreases monotonically to $\mathbf{0}$ as $k \rightarrow \infty$, there is an increasing sequence $k_{n}$ of non-negative integers such that $a_{n k_{n}} \rightarrow \mathbf{0}$.

Hence, defining the closure $\bar{A}$ of a subset $A$ of $\mathbb{B}$ to be the collection of all limits of strongly converging sequences of members of $A$, we get a closure operator and the corresponding sequential topology of $\mathbb{B}$. In [11], Maharam shows that a $\sigma$-algebra $\mathbb{B}$ supports a strictly positive continuous submeasure if and only if its sequential topology is metrizable (in which case $\mathbb{B}$ becomes a topological group under the relation of symmetric difference). This shows clearly the utmost importance of the sequential topology in the study of von Neumann's problem. As correctly realized in [11], the metrizability of the sequential topology of $\mathbb{B}$ is captured by the algebraic (or better to say, combinatorial) properties of the ideal of all countable subsets of $\mathbb{B}$ which do not contain $\mathbf{0}$ in their closures. In fact, it turns out that it is more advantageous to work with the orthogonal of this ideal. Thus, let $\mathcal{I}_{\mathbb{B}}$ be the collection of all countable subsets $A$ of $\mathbb{B}^{+}$for which we can find a maximal cellular family $\mathcal{C}$ of $\mathbb{B}$ such that

$$
c \uparrow A=\{a \in A: c \cdot a \neq \mathbf{0}\}
$$

is finite for all $c \in \mathcal{C}$. Note that if a maximal cellular family $\mathcal{C}$ witnesses the membership of $A$ in $\mathcal{I}_{\mathbb{B}}$ then so does any other maximal cellular family $\mathcal{D}$ which refines $\mathcal{C}$, i.e., has the property that every $d$ from $\mathcal{D}$ is included in a (necessarily unique) member of $\mathcal{C}$. It follows that $\mathcal{I}_{\mathbb{B}}$ is indeed an ideal of subsets of $\mathbb{B}^{+}$, i.e., it is closed under unions, since for every pair $\mathcal{C}_{0}$ and $\mathcal{C}_{1}$ of maximal cellular families of $\mathbb{B}$ there is a maximal cellular family $\mathcal{D}$ of $\mathbb{B}$ which refines both. The ideal $\mathcal{I}_{\mathbb{B}}$ appears for the first time in [1] for $\mathbb{B}$ a Suslin algebra. The $\sigma$-distributivity of the Suslin algebra is used in [1] to show that $\mathcal{I}_{\mathbb{B}}$ is a $P$-ideal, i.e. it has the following weak form of $\sigma$-completeness: For every sequence $A_{n}$ of members of $\mathcal{I}_{\mathbb{B}}$ there is a member $B$ of $\mathcal{I}_{\mathbb{B}}$ such that $A_{n} \backslash B$ is finite for all $n$. To see this, for each $n$, fix a maximal cellular family 
$\mathcal{C}_{n}$ witnessing $A_{n} \in \mathcal{I}_{\mathbb{B}}$. Using $\sigma$-distributivity of $\mathbb{B}$ we can find a maximal cellular family $\mathcal{C}$ of $\mathbb{B}$ which refines $\mathcal{C}_{n}$ for all $n$, and therefore witnesses simultaneously $A_{n} \in \mathcal{I}_{\mathbb{B}}$ for all $n$. Since $\mathbb{B}$ satisfies the countable chain condition, $\mathcal{C}$ is countable, so we can enumerate it in a simple sequence $c_{k}$. Let

$$
B=\bigcup_{n} A_{n} \backslash\left(\bigcup_{k<n} c_{k} \uparrow A_{n}\right) .
$$

Clearly, this $B$ almost includes $A_{n}$ for every $n$. Note that the same argument applies if the cellular family $\mathcal{C}$ only almost refines each of the families $\mathcal{C}_{n}$ in the sense that for each $n$ every element of $\mathcal{C}$ intersects only finitely many members of $\mathcal{C}_{n}$. Note also that the existence of such a $\mathcal{C}$ is guaranteed by the weak distributivity of $\mathbb{B}$. This shows the following useful fact.

Lemma 1. The ideal $\mathcal{I}_{\mathbb{B}}$ is a P-ideal for every weakly distributive $\sigma$ algebra $\mathbb{B}$ satisfying the countable chain condition.

This fact has been first put out in print by Quickert [16] while analyzing a problem of Prikry that is quite closely related to that of von Neumann [14] (see also [22]).

Back to the sequential topology of $\mathbb{B}$, note that if $A$ is a countably infinite subset of $\mathbb{B}^{+}$then $A$ belongs to $\mathcal{I}_{\mathbb{B}}$ if and only if for some (all) one-to-one enumerations $a_{n}$ of $A$ the sequence $a_{n}$ converges to $\mathbf{0}$, i.e., $\lim \sup a_{n}=\mathbf{0}$. So the orthogonal of $\mathcal{I}_{\mathbb{B}}$ is

$$
\mathcal{I}_{\mathbb{B}}^{\perp}=\left\{X \subseteq \mathbb{B}: X \text { is countable and } X \cap A \text { is finite for all } A \in \mathcal{I}_{\mathbb{B}}\right\} .
$$

More generally, we say that an arbitrary subset $X$ of $\mathbb{B}^{+}$is orthogonal to $\mathcal{I}_{\mathbb{B}}$ and write $X \perp \mathcal{I}_{\mathbb{B}}$ if $X$ has a finite intersection with every member of $\mathcal{I}_{\mathbb{B}}$. Thus, $\mathcal{I}_{\mathbb{B}}^{\perp}$ is simply the collection of all countable sets that are orthogonal to $\mathcal{I}_{\mathbb{B}}$. Put even more simply, a set is orthogonal to $\mathcal{I}_{\mathbb{B}}$ if its closure misses $\mathbf{0}$. We say that $\mathcal{I}_{\mathbb{B}}^{\perp}$ is countably generated if there is a sequence $X_{n}$ of subsets of $\mathbb{B}^{+}$that are orthogonal to $\mathcal{I}_{\mathbb{B}}$ with the property that every member of $\mathcal{I}_{\mathbb{B}}^{\perp}$ is included modulo a finite set in some member of the sequence. The following result of Maharam [11] explains our interest in these notions.

Lemma 2 ([11]). A $\sigma$-algebra $\mathbb{B}$ supports a strictly positive continuous submeasure if and only if the orthogonal of $\mathcal{I}_{\mathbb{B}}$ is countably generated.

Proof. For the convenience of the reader we sketch the argument of the reverse implication as it appears in [11] though in a slightly different terminology and with one unnecessary assumption. First of all note that if $\mathcal{I}_{\mathbb{B}}^{\perp}$ is countably generated then $\mathbb{B}$ satisfies the countable chain condition as well as the diagonal sequence principle (DS). So we can choose a sequence $X_{n}$ of subsets of $\mathbb{B}^{+}$which is increasing in $n$ and consists of sets that are at the same time upwards closed and closed in the sequential topology. As already pointed out, the corresponding sequence $V_{n}$ of complements consists of 
neighborhoods of $\mathbf{0}$ that decrease in $n$ and are downwards closed in the natural ordering of $\mathbb{B}$. Note that any subset of $\mathbb{B}$ whose closure does not contain $\mathbf{0}$ must be included in some $X_{n}$. It follows that the $V_{n}$ form a countable neighborhood base of $\mathbf{0}$. Note also that the operations $\vee$ and $\triangle$ are continuous at $(\mathbf{0 , 0})$ or else we would, say, be able to find a neighborhood $U$ of $\mathbf{0}$ and two sequences $a_{n}$ and $b_{n}$ such that $a_{n}, b_{n} \in V_{n}$ but $a_{n} \vee b_{n}$ is not in $U$. It follows that $\limsup a_{n}=\mathbf{0}$ and $\lim \sup b_{n}=\mathbf{0}$ while $\lim \sup a_{n} \vee b_{n} \neq \mathbf{0}$, a contradiction. It follows that $\mathbb{B}$ with the symmetric difference as the group operation is a metrizable abelian group so it has a bounded invariant metric $\varrho$. Then $\nu(b)=$ $\sup \{\varrho(\mathbf{0}, a): a \leq b\}$ defines a strictly positive continuous submeasure on $\mathbb{B}$.

Note that in the case when $\mathcal{I}_{\mathbb{B}}^{\perp}$ is countably generated one can cover $\mathbb{B}^{+}$ by countably many sets that are orthogonal to $\mathcal{I}_{\mathbb{B}}$. It is actually this condition that forms a part of a general dichotomy for P-ideals considered in [21]. For general P-ideals $\mathcal{I}$ of countable subsets of some set $S$ the alternative that the set $S$ can be covered by countably many subsets that are orthogonal to $\mathcal{I}$ is unlikely to imply that $\mathcal{I}^{\perp}$ is countably generated. Therefore, the following result of Balcar, Jech, and Pazák [3] (based on a previous work from [2]) came as a surprise.

LEMMA 3 ([3]). The following are equivalent for a weakly distributive $\sigma$-algebra satisfying the countable chain condition:

(1) The orthogonal of $\mathcal{I}_{\mathbb{B}}$ is countably generated.

(2) $\mathbb{B}^{+}$can be decomposed into countably many subsets orthogonal to $\mathcal{I}_{\mathbb{B}}$.

Proof. For the convenience of the reader again, we sketch the arguments as they appear in [3] and [2] though in a different terminology. Only the implication from (2) to (1) requires an argument. So, let $X_{n}$ be a sequence of subsets of $\mathbb{B}^{+}$which cover $\mathbb{B}^{+}$and which are orthogonal to $\mathcal{I}_{\mathbb{B}}$. Again we may assume that $X_{n}$ increase with $n$ and that they are upwards closed as well as closed in the sequential topology of $\mathbb{B}$. Hence the corresponding sequence $V_{n}$ of complements is a sequence of open neighborhoods that are downwards closed under the ordering of $\mathbb{B}$.

First of all note that $\bigcap_{n} \bar{V}_{n}=\{\mathbf{0}\}$. For suppose there is a non-zero member $b$ in that intersection. Then for each $n$ we can find a sequence $a_{n k}$ of members of $V_{n}$ which strongly converges to $b$ as $k \rightarrow \infty$. By (DS) we can find a diagonal sequence $a_{n k_{n}}$ such that $a_{n k_{n}} \rightarrow b$. So there must be an $m$ such that $c=\bigwedge_{n>m} a_{n k_{n}} \neq \mathbf{0}$. Pick an $n>m$ such that $c$ does not belong to $V_{n}$. Then $c \leq a_{n k_{n}} \in V_{n}$, contradicting the downward closure of $V_{n}$.

Note that this argument also shows that every sequence $a_{n}$ such that $a_{n} \vee a_{n+1} \vee \cdots \vee a_{n+k} \in V_{n}$ for all $n$ and $k$ must converge to $\mathbf{0}$. So, as in the proof of Lemma 2, one shows that the operation $\vee$ (and therefore $\triangle$ ) is continuous at $(\mathbf{0}, \mathbf{0})$ and so, in particular, the sequential topology of $\mathcal{B}$ is regular. 
Now we claim that the $V_{n}$ form a neighborhood base at $\mathbf{0}$, which is of course just another formulation of (1). Otherwise, fixing a closed neighborhood $U$ of $\mathbf{0}$ not refined by any $V_{n}$ and using the fact that the boolean operation $\vee$ is separately continuous, we can build a sequence $a_{n}$ such that $\bigvee_{k=n}^{m} a_{k} \in V_{n} \backslash U$ for all $n \leq m$. It follows that $\bigvee_{k>n} a_{k} \in \bar{V}_{n} \backslash U$ for all $n$. Since the $\bar{V}_{n}$ have only the point $\mathbf{0}$ in their intersection, we conclude that $\lim \sup a_{n}=\mathbf{0}$. This shows that $a_{n} \rightarrow \mathbf{0}$. So there must be an $n$ such that $a_{n} \in U$, a contradiction.

It follows that a weakly distributive $\sigma$-algebra $\mathbb{B}$ satisfying the countable chain condition supports a strictly positive continuous submeasure if and only if $\mathbb{B}^{+}$can be decomposed into countably many subsets orthogonal to $\mathcal{I}_{\mathbb{B}}$ if and only if $\mathbf{0}$ is a $G_{\delta}$-point in $\mathbb{B}$ with the sequential topology.

The relationship between the weak distributivity and P-ideals becomes even more striking if one examines the separable case of the (sub)measurability problem. The separability refers to the metric one defines from a continuous submeasure which in the case the submeasure is missing has its simple algebraic formulation as a condition on the way one can generate the algebra. Recall that a complete boolean algebra $\mathbb{B}$ is countably generated if there is a countable subset $\mathbb{B}_{0}$ of $\mathbb{B}$ such that every complete subalgebra of $\mathbb{B}$ which contains $\mathbb{B}_{0}$ must be equal to $\mathbb{B}$. By the Sikorski-Loomis theorem every countably generated $\sigma$-algebra $\mathbb{B}$ is isomorphic to a quotient algebra of the form $\operatorname{Borel}\left(2^{\mathbb{N}}\right) / \mathcal{I}$ for some Borel-generated $\sigma$-ideal $\mathcal{I}$ in $2^{\mathbb{N}}$. As pointed out in [23], the homomorphism extension theorem of Matthes (see [12], [13], [17]) gives a considerable information about the $\sigma$-ideal $\mathcal{I}$ in the case $\mathbb{B}$ is a weakly distributive $\sigma$-algebra satisfying the countable chain condition. To describe this, following [23], let us say that a $\sigma$-ideal $\mathcal{I}$ on $2^{\mathbb{N}}$ is regular if it satisfies the following conditions:

(a) $\mathcal{I}$ is uniform (i.e., contains all singletons) and proper (i.e., it does not contain the whole set $2^{\mathbb{N}}$ ).

(b) $\mathcal{I}$ is generated by the family of $G_{\delta}$-sets that belong to $\mathcal{I}$.

(c) $\mathcal{I}$ satisfies the countable chain condition (i.e., every family of disjoint $\mathcal{I}$-positive Borel sets must be countable).

(d) $\mathcal{I}$ is inner regular relative to the family of compact sets (i.e., every $\mathcal{I}$-positive Borel set contains an $\mathcal{I}$-positive compact subset).

(e) For every sequence $\left(\mathcal{G}_{i}\right)$ of countable downwards directed families of open subsets of $2^{\mathbb{N}}$ with the property that $\bigcap \mathcal{G}_{i} \in \mathcal{I}$ for all $i$ there is a sequence $\left(G_{i}^{k}\right) \subseteq \mathcal{G}_{i}$ for each $i$ such that $\bigcap_{k} \bigcup_{i} G_{i}^{k} \in \mathcal{I}$.

In [23, pp. 272-276], the following consequence of the homomorphism extension theorem of Matthes has been explicitly pointed out. 
LEMMA 4. An atomless countably generated $\sigma$-algebra is weakly distributive and satisfies the countable chain condition if and only if is isomorphic to a quotient algebra of the form $\operatorname{Borel}\left(2^{\mathbb{N}}\right) / \mathcal{I}$ for some regular $\sigma$-ideal $\mathcal{I}$ on $2^{\mathbb{N}}$.

From Theorem 1 and Lemma 4 we obtain the following result which characterizes regular $\sigma$-ideals arising from continuous outer-measures on $2^{\mathbb{N}}$.

THEOREM 2. The following are equivalent for a Borel-generated $\sigma$-ideal $\mathcal{I}$ on $2^{\mathbb{N}}$ :

(1) $\mathcal{I}$ is the $\sigma$-ideal of null sets for some normalized uniform diffused and continuous outer measure on $2^{\mathbb{N}}$.

(2) $\mathcal{I}$ is regular and its quotient algebra $\operatorname{Borel}\left(2^{\mathbb{N}}\right) / \mathcal{I}$ satisfies the $\sigma$-finite chain condition.

In [18], Solecki gives a characterization of $\sigma$-ideals arising from continuous outer measures on $2^{\mathbb{N}}$ in terms of P-ideals of subsets of the tree $2^{<\mathbb{N}}$ of finite binary sequences. It turns out that all regular $\sigma$-ideals on $2^{\mathbb{N}}$ are expressible in terms of P-ideals on $2^{<\mathbb{N}}$. To see this we need the following piece of notation for a subset $A$ of $2^{<\mathbb{N}}$ :

$$
[A]=\left\{x \in 2^{\mathbb{N}}: \exists^{\infty} k x\lceil k \in A\} .\right.
$$

For a family $\mathcal{J}$ of subsets of $2^{<\mathbb{N}}$ let $[\mathcal{J}]$ be the downward closure of the family

$$
\{[A]: A \in \mathcal{J}\}
$$

Then we have the following description of regular $\sigma$-ideals on $2^{\mathbb{N}}$.

LEMMA 5. Every regular $\sigma$-ideal $\mathcal{I}$ on $2^{\mathbb{N}}$ has the form $[\mathcal{J}]$ for some $P$-ideal $\mathcal{J}$ on $2^{<\mathbb{N}}$.

Proof. For a compact subset $K$ of $2^{\mathbb{N}}$, let

$$
T_{K}=\{x\lceil k: x \in K, k \in \mathbb{N}\}
$$

denote the subtree of $2^{<\mathbb{N}}$ such that $\left[T_{K}\right]=K$. Let $\mathcal{J}$ be the collection of all subsets $A$ of $2^{<\mathbb{N}}$ for which one can find a maximal family $\mathcal{K}$ of pairwise disjoint $\mathcal{I}$-positive sets such that $A \cap T_{K}$ is finite for all $K \in \mathcal{K}$. Since $\mathcal{I}$ is a regular $\sigma$-ideal, the quotient algebra $\operatorname{Borel}\left(2^{\mathbb{N}}\right) / \mathcal{I}$ is weakly distributive and compact sets are dense, so working as in the proof of Lemma 1 one concludes that $\mathcal{J}$ is a P-ideal of subsets of $2^{<\mathbb{N}}$.

We claim that $\mathcal{I}=[\mathcal{J}]$. First of all, note that for $A \in \mathcal{J}$ the $G_{\delta}$-set $[A]$ cannot contain any $\mathcal{I}$-positive compact subset, so by property (d) of $\mathcal{I}$ it must belong to $\mathcal{I}$. Conversely, consider an arbitrary $G_{\delta^{-s e t}} G \in \mathcal{I}$. Choose a maximal family $\mathcal{K}$ of pairwise disjoint $\mathcal{I}$-positive sets each of which is disjoint from $G$. List $\mathcal{K}$ as a simple sequence $\left(K_{i}\right)$ and then represent $G$ as intersection of a decreasing sequence $\left(G_{k}\right)$ of open sets such that $G_{k} \cap K_{i}=\emptyset$ 
whenever $i \leq k$. Now, find a sequence $A_{k}$ of antichains of the complete binary tree $2^{<\mathbb{N}}$ such that:

(1) $A_{k}$ refines $A_{i}$ and they are disjoint whenever $i<k$,

(2) $A_{k} \cap T_{K_{i}}=\emptyset$ whenever $i \leq k$,

(3) $G_{k}=\left\{x \in 2^{\mathbb{N}}: \exists n x\left\lceil n \in A_{k}\right\}\right.$ for all $k$.

For each $i$ find a maximal family $\mathcal{H}_{i}$ of pairwise disjoint compact $\mathcal{I}$-positive subsets of $K_{i}$ such that for every $H \in \mathcal{H}$ and every $k<i$, either $H \cap G_{k}=\emptyset$, or else there is $s \in A_{k}$ such that every element of $H$ extends $s$. Let $\mathcal{H}=\bigcup_{i} \mathcal{H}_{i}$ and let $A=\bigcup_{k} A_{k}$. Then $\mathcal{H}$ is a maximal family of pairwise disjoint $\mathcal{I}$ positive compact sets such that $T_{H} \cap A$ is finite for all $H \in \mathcal{H}$. It follows that $A$ belongs to $\mathcal{J}$ and therefore $G=[A] \in[\mathcal{J}]$. This finishes the proof.

It follows that every atomless countably generated weakly distributive $\sigma$-algebra $\mathcal{B}$ with the countable chain condition has the form $\operatorname{Borel}\left(2^{\mathbb{N}}\right) /[\mathcal{J}]$ for some P-ideal $\mathcal{J}$ of subsets of the complete binary tree $2^{<\mathbb{N}}$. So it remains to be investigated which P-ideals on $2^{<\mathbb{N}}$ correspond to algebras $\mathcal{B}$ that support a strictly positive continuous submeasure and which correspond to algebras $\mathcal{B}$ supporting a strictly positive countably additive measure. Some work on this has already been done in [18] and [4], where it is shown that in Theorem 2 some definability assumptions on $\mathcal{I}=[\mathcal{J}]$ could replace the chain condition restriction on the quotient algebra $\operatorname{Borel}\left(2^{\mathbb{N}}\right) / \mathcal{I}$.

3. Proof of Theorem 1. It has already been observed that (1) implies (2) so we concentrate on proving that (2) implies (1). Thus, we start with a weakly distributive $\sigma$-algebra $\mathbb{B}$ satisfying the countable chain condition. Let $\mathcal{I}=\mathcal{I}_{\mathbb{B}}$ be the corresponding P-ideal, i.e.,

$$
\mathcal{I}_{\mathbb{B}}=\left\{A \in\left[\mathbb{B}^{+}\right]^{\leq \omega}: \exists \mathcal{C} \in \mathcal{M C}(\mathbb{B}) \forall c \in \mathcal{C} c \mid A \text { is finite }\right\},
$$

where $\mathcal{M C}(\mathbb{B})$ denotes the collection of all maximal cellular families of elements of $\mathbb{B}^{+}$. We shall show that if $\mathbb{B}^{+}$cannot be decomposed into countably many sets each orthogonal to $\mathcal{I}$ then the $\sigma$-algebra $\mathbb{B}$ does not satisfy the stronger $\sigma$-finite chain condition of Horn and Tarski. Then the proof is completed by using Lemmas 2 and 3 above. So from now on we assume that $\mathbb{B}^{+}$cannot be covered by countably many sets orthogonal to $\mathcal{I}$ and work for showing that $\mathbb{B}$ fails to satisfy the $\sigma$-finite chain condition. So, let

$$
\mathbb{B}^{+}=\bigcup_{k=0}^{\infty} \mathbb{B}_{k}
$$

be a given decomposition and work towards showing that there must be some $\mathbb{B}_{k}$ containing an infinite cellular family. This will conclude our proof of Theorem 1. 
Following [21], we let $\mathcal{P}$ be the collection of all pairs $p=\left\langle x_{p}, \mathfrak{X}_{p}\right\rangle$ such that:

(i) $x_{p}$ is an element of $\mathcal{I}$,

(ii) $\mathfrak{X}_{p}$ is a countable collection of cofinal subsets of $\left\langle\mathcal{I}, \subseteq^{*}\right\rangle$.

We order $\mathcal{P}$ by letting $q \geq p$ and saying that $q$ extends $p$ whenever:

(iii) $x_{p} \subseteq x_{q}$,

(iv) $\mathfrak{X}_{p} \subseteq \mathfrak{X}_{q}$,

(v) for every $X \in \mathfrak{X}_{p}$ the set $\left\{x \in X: x_{q} \backslash x_{p} \subseteq x\right\}$ is $\subseteq^{*}$-cofinal in $\mathcal{I}$ and it belongs to the family $\mathfrak{X}_{q}$.

Lemma 6. For every $p \in \mathcal{P}$ and every maximal cellular family $\mathcal{C}$ of $\mathbb{B}^{+}$ there is $q \geq p$ such that some member of $x_{q} \backslash x_{p}$ refines a member of $\mathcal{C}$.

Proof. Let $\mathbb{B}^{+}\left\lceil\mathcal{C}\right.$ be the collection of all $b \in \mathbb{B}^{+}$which refine a member of $\mathcal{C}$. Then $\mathbb{B}^{+} \uparrow \mathcal{C}$ is coinitial in $\mathbb{B}^{+}$so by our assumption it cannot be covered by countably many sets orthogonal to $\mathcal{I}$. Suppose the conclusion of the lemma fails. Then for every member $b \in\left(\mathbb{B}^{+}\lceil\mathcal{C}) \backslash x_{p}\right.$ there is $X \in \mathfrak{X}_{p}$ such that

$$
X(b)=\{x \in X: b \in x\}
$$

is not cofinal in $\left\langle\mathcal{I}, \subseteq^{*}\right\rangle$. For $X \in \mathfrak{X}_{p}$, let

$$
\mathcal{B}(X)=\left\{b \in\left(\mathbb{B}^{+}\lceil\mathcal{C}) \backslash x_{p}: X(b) \text { is not cofinal in }\left\langle\mathcal{I}, \subseteq^{*}\right\rangle\right\} .\right.
$$

By our assumption $\left\{\mathcal{B}(X): X \in \mathfrak{X}_{p}\right\}$ is a countable collection of subsets of $\mathcal{B}^{+}$which covers $\left(\mathbb{B}^{+}\lceil\mathcal{C}) \backslash x_{p}\right.$. So we shall reach a contradiction the moment we show that each $\mathcal{B}(X)$ is orthogonal to $\mathcal{I}$. For suppose that for some $X \in \mathfrak{X}_{p}$ there is an infinite subset $z$ of $\mathcal{B}(X)$ belonging to $\mathcal{I}$. Since $\mathcal{I}$ is a P-ideal, removing a finite subset of $z$, we may assume that

$$
\mathcal{Y}=\{y \in X: z \subseteq y\}
$$

is cofinal in $\left\langle\mathcal{I}, \subseteq^{*}\right\rangle$. Choose an arbitrary $b \in z$. Then $b \in \mathcal{B}(X)$ and $\mathcal{Y} \subseteq X(b)$ and therefore $X(b)$ is also cofinal in $\left\langle\mathcal{I}, \subseteq^{*}\right\rangle$. But this is a contradiction since, by the definition of $\mathcal{B}(X)$ and the fact that $b \in \mathcal{B}(X)$, the set $X(b)$ is not supposed to be cofinal in $\left\langle\mathcal{I}, \subseteq^{*}\right\rangle$.

Choose a countable elementary substructure $M$ of some large enough structure of the form $\left\langle H_{\theta}, \in\right\rangle$ in such a way that the substructure $M$ contains as elements all the relevant objects accumulated so far.

Lemma 7. Suppose $p \in \mathcal{P} \cap M$ and $z_{M} \in \mathcal{I}$ is such that $z_{M} \subseteq M \cap \mathbb{B}^{+}$ and $x \subseteq \subseteq^{*} z_{M}$ for all $x \in \mathcal{I} \cap M$. Then for every $k \in \mathbb{N}$ there is $q \geq p$ in $\mathcal{P} \cap M$ such that $x_{q} \backslash x_{p} \subseteq z_{M}$ and such that either

(a) $x_{q} \backslash x_{p}$ contains a member of $\mathbb{B}_{k}$, or

(b) there is no $r \geq q$ in $\mathcal{P}$ for which $x_{r} \backslash x_{p}$ contains a member of $\mathbb{B}_{k}$. 
Proof. Suppose such a $q$ cannot be found. Let $\mathcal{D}$ be the collection of all $q \in \mathcal{P}$ satisfying (a) or (b). Then $\mathcal{D} \in M$ and $\mathcal{D}$ is dense above $p$ in the sense that for every $q \geq p$ there is $r \geq q$ such that $r \in \mathcal{D}$. Let

$$
Y_{0}=\left\{y \in \mathcal{I}: \exists F \in[y]^{<\omega} \forall q \geq p\left(q \in \mathcal{D} \rightarrow x_{q} \backslash x_{p} \not \subset y \backslash F\right)\right\} .
$$

Clearly $Y_{0} \in M$. Note that every member $y$ of $\mathcal{I} \cap M$ belongs to $Y_{0}$ since the finite set $y \backslash z_{M}$ witnesses this by our assumption that there is no $q \geq p$ satisfying the conclusion of the lemma. By elementarity of $M$, it follows that $Y_{0}$ is actually equal to $\mathcal{I}$. Fix a mapping $A \mapsto z_{A}$ from $[\mathcal{I}]^{\omega}$ into $\mathcal{I}$ belonging to $M$ and having the property that

(vi) $z_{A} \subseteq \bigcup A$ and $x \subseteq^{*} z_{A}$ for every $x \in A$.

Then by the fact that $Y_{0}=\mathcal{I} \supseteq\left\{z_{A}: A \in[\mathcal{I}]^{\omega}\right\}$ for each $A \in[\mathcal{I}]^{\omega}$, we can fix a finite set $F_{A} \subseteq z_{A}$ such that

$$
\text { (vii) } \forall q \geq p\left(q \in \mathcal{D} \rightarrow x_{q} \backslash x_{p} \nsubseteq z_{A} \backslash F_{A}\right) \text {. }
$$

Moreover we may assume that the mapping $A \mapsto F_{A}$ belongs to $M$. Note that this map can be viewed as a finite sequence of regressive maps on the stationary domain $[\mathcal{I}]^{\omega}$, so the corresponding analogue of the Pressing Down Lemma applies. Hence, we can find, in $M$, a stationary subset $S$ of $[\mathcal{I}]^{\omega}$ and a finite set $F$ such that $F_{A}=F$ for all $A \in S$. Let

$$
p_{1}=\left\langle x_{p}, \mathfrak{X}_{p} \cup\left\{z_{A} \backslash F: A \in S\right\}\right\rangle .
$$

Then $p_{1} \in \mathcal{P} \cap M$ and $p_{1} \geq p$. Since $\mathcal{D}$ is dense above $p$ and since $M$ is an elementary substructure of $H_{\theta}$, there is $q \in \mathcal{D} \cap M$ such that $q \geq p_{1}$. By the definition of the ordering $\geq$ of $\mathcal{P}$, there exist cofinally many members of the family $\left\{z_{A} \backslash F: A \in S\right\}$ that contain the set $x_{q} \backslash x_{p}$. In other words, we can find an $A \in S$ so that

(viii) $x_{q} \backslash x_{p} \subseteq z_{A} \backslash F$.

On the other hand, from the fact that $A$ belongs to $S$, we conclude that $F=F_{A}$, leading to something that contradicts (vii). This finishes the proof of Lemma 7.

A similar argument proves the following lemma.

Lemma 8. Suppose $p \in \mathcal{P} \cap M$ and let $z_{M} \in \mathcal{I}$ be such that $z_{M} \subseteq M \cap \mathbb{B}^{+}$ and $x \subseteq^{*} z_{M}$ for all $x \in \mathcal{I} \cap M$. Then for every finite subset $F$ of $\mathbb{B}^{+} \cap M$, every $p \in \mathcal{P} \cap M$ and every $k \in \mathbb{N}$ there is $q \geq p$ in $M \cap \mathcal{P}$ such that $x_{q} \backslash x_{p} \subseteq z_{M}$ and such that either

(a) $x_{q} \backslash x_{p}$ contains a member $b$ of $\mathbb{B}_{k}$ such that $b \wedge a=\mathbf{0}$ for every $a \in F$, or

(b) there is no $r \geq q$ in $\mathcal{P}$ such that $x_{r} \backslash x_{p}$ contains a member $b$ of $\mathbb{B}_{k}$ with $b \wedge a=\mathbf{0}$ for every $a \in F$. 
Working in $M$ and starting from $p_{0}=\langle\emptyset,\{\mathcal{I}\}\rangle$ we build a decreasing sequence $p_{n}=\left\langle x_{n}, \mathfrak{X}_{n}\right\rangle(n \in \mathbb{N})$ of members of $\mathcal{P} \cap M$ and a $\subseteq$-decreasing sequence $z_{n}(n \in \mathbb{N})$ of members of $\mathcal{I}$ such that

(ix) $\forall n \in \mathbb{N} \forall x \in \mathcal{I} \cap M x \subseteq^{*} z_{n}$,

(x) $\forall n \in \mathbb{N} x_{n+1} \backslash x_{n} \subseteq z_{n}$.

We choose $z_{0}$ to be an arbitrary member of $\mathcal{I}$ such that $z_{0} \subseteq M \cap \mathbb{B}^{+}$and $x \subseteq^{*} z_{0}$ for all $x \in \mathcal{I} \cap M$. We will also ensure that the set $z_{n+1}$ is obtained from $z_{n}$ by removing from it only a finite subset so (ix) will automatically remain satisfied. The recursive construction is done in the following manner. Via some book-keeping device, we associate to each $n$ and each $X \in \mathfrak{X}_{n}$ an integer $m \geq n$ where we perform the following procedure. We first let

$$
X_{1}=\left\{x \in X: x_{m} \backslash x_{n} \subseteq x\right\} .
$$

Then we know that $X_{1} \in M$ and that $X_{1}$ is cofinal in $\left\langle\mathcal{I}, \subseteq^{*}\right\rangle$. So there is a finite set $F_{m} \subseteq z_{m}$ such that

$$
X_{2}=\left\{x \in X_{1}: z_{m} \backslash F_{m} \subseteq x\right\}
$$

is still cofinal in $\left\langle\mathcal{I}, \subseteq^{*}\right\rangle$. We let $z_{m+1}=z_{m} \backslash F_{m}$ and

$$
p_{m+1}=\left\langle x_{m}, \mathfrak{X}_{m} \cup\left\{x \backslash F_{m}: x \in \mathcal{I}\right\}\right\rangle .
$$

This together with $(\mathrm{x})$ ensures that

$$
\text { (xi) } \forall l>m\left(x_{l} \backslash x_{m}\right) \cap F_{m}=\emptyset \text {. }
$$

and therefore that

$$
\text { (xii) } \forall x \in X_{2} x_{\omega} \backslash x_{n} \subseteq x,
$$

where $x_{\omega}=\bigcup_{i=0}^{\infty} x_{i}$. This will ensure that if we let

$$
\mathfrak{X}_{\omega}=\left(\bigcup_{i=0}^{\infty} \mathfrak{X}_{i}\right) \cup\left\{\left\{x \in X: x_{\omega} \backslash x_{n} \subseteq x\right\}: n \in \mathbb{N}, X \in \mathfrak{X}_{n}\right\}
$$

then $p_{\omega}=\left\langle x_{\omega}, \mathfrak{X}_{\omega}\right\rangle$ is a member of $\mathcal{P}$ extending $p_{n}$ for all $n$. Using Lemmas 7 and 8 we can arrange our book-keeping device so that the following two conditions are also satisfied:

(xiii) For every $k, n \in \mathbb{N}$ there is $m \geq n$ such that either

$$
\left(x_{m+1} \backslash x_{m}\right) \cap \mathbb{B}_{k} \neq \emptyset \text {, }
$$

or

$$
\forall q \geq p_{m+1} \quad\left(x_{q} \backslash x_{m}\right) \cap \mathbb{B}_{k}=\emptyset .
$$

(xiv) For every $k, n \in \mathbb{N}$, for every $F \in\left[x_{n}\right]^{<\omega}$ there is $m \geq n$ such that either

$$
\exists b \in\left(x_{m+1} \backslash x_{m}\right) \cap \mathbb{B}_{k} \forall a \in F \quad(b \cdot a=0),
$$


or

$$
\forall q \geq p_{m+1} \forall b \in\left(x_{q} \backslash x_{m}\right) \cap \mathbb{B}_{k} \exists a \in F \quad(b \cdot a \neq 0) .
$$

This finishes our description of the recursive construction. From the facts $p_{\omega} \in \mathcal{P}$ and $p_{\omega} \geq p_{0}$ we conclude that, in particular, $x_{\omega} \in \mathcal{I}$. Applying the definition of the $\mathrm{P}$-ideal $\mathcal{I}=\mathcal{I}_{\mathcal{B}}$ to the set $x_{\omega}$, we conclude that there exists a maximal cellular family $\mathcal{C}$ of $\mathbb{B}^{+}$such that

$(\mathrm{xv}) \forall c \in \mathcal{C}\left\{a \in x_{\omega}: c \wedge a>\mathbf{0}\right\}$ is finite.

Applying Lemma 6 to $p_{\omega}$ and $\mathcal{C}$, we find an extension $q \geq p_{\omega}$ such that $x_{q} \backslash x_{\omega}$ contains a member $b_{0}$ which refines some member of the cellular family $\mathcal{C}$. Fix $k_{0} \in \mathbb{N}$ such that $b_{0} \in \mathbb{B}_{k_{0}}$. From (xiii), we infer that

$$
\text { (xvi) } \exists^{\infty} m\left(x_{m+1} \backslash x_{m}\right) \cap \mathbb{B}_{k_{0}} \neq \emptyset \text {. }
$$

By $(\mathrm{xv})$ we can find an integer $\bar{m}$ such that

$$
\text { (xvii) } \forall m>\bar{m} \forall a \in\left(x_{m} \backslash x_{\bar{m}}\right)\left(b_{0} \cdot a=0\right) \text {. }
$$

Pick $m_{0}>\bar{m}$ for which we can find $a_{0} \in x_{m_{0}+1} \backslash x_{m_{0}}$ belonging to $\mathbb{B}_{k_{0}}$ (see (xvi)). Applying (xiv) to $k=k_{0}, n=m_{0}+1$ and $F=\left\{a_{0}\right\}$ and noticing (see (xvii)) that the second alternative fails, we get $m_{1} \geq m_{0}+1$ and $a_{1} \in\left(x_{m_{1}+1} \backslash x_{m_{1}}\right) \cap \mathbb{B}_{k_{0}}$ such that $a_{0} \cdot a_{1}=0$. Applying (xvii) again, we get

$$
\text { (xviii) } \forall a \in\left\{a_{0}, a_{1}\right\}\left(b_{0} \cdot a=0\right) \text {. }
$$

Applying (xiv) again but now for $k=k_{0}, n=m_{1}+1$ and $F=\left\{a_{0}, a_{1}\right\}$, we get an $m_{2}>m_{1}$ satisfying one of its two alternatives, the second of which contradicts (xviii). This gives us an $a_{2} \in\left(x_{m_{2}+1} \backslash x_{m_{2}}\right) \cap \mathbb{B}_{k_{0}}$ such that

$$
a_{2} \cdot a_{0}=0=a_{2} \cdot a_{1},
$$

so one can continue by applying (xiv) to $k=k_{0}, n=m_{2}+1$ and $F=$ $\left\{a_{0}, a_{1}, a_{2}\right\}$, and so on. It is therefore clear that we can continue this procedure indefinitely and produce an infinite cellular family

$$
\left\{a_{0}, a_{1}, a_{2}, \ldots\right\} \subseteq \mathbb{B}_{k_{0}}
$$

completing the task put forward at the beginning of this section. As indicated in the same place the completion of this task finishes the proof of Theorem 1.

\section{References}

[1] U. Abraham and S. Todorcevic, Partition properties of $\omega_{1}$ compatible with $\mathrm{CH}$, Fund. Math. 152 (1997), 165-181.

[2] B. Balcar, W. Główczyński and T. Jech, The sequential topology on complete Boolean algebras, ibid. 155 (1998), 27-41. 
[3] B. Balcar, T. Jech and T. Pazák, Complete ccc Boolean algebras, the order sequential topology and a problem of von Neumann, preprint, ArXiv 28/12/2003.

[4] I. Farah and J. Zapletal, Between Maharam's and von Neumann's problems, preprint, 2004.

[5] M. Fréchet, Sur la notion de voisinage dans les ensembles abstraits, Bull. Sci. Math. 42 (1918), 138-156.

[6] D. H. Fremlin, Chapter 39: Measurable algebras, in: Measure Theory, Vol. 3, Torres Fremlin, 2002.

[7] H. Gaifman, Concerning measures on Boolean algebras, Pacific J. Math. 14 (1964), $61-73$.

[8] A. Horn and A. Tarski, Measures in Boolean algebras, Trans. Amer. Math. Soc. 64 (1948), 467-497.

[9] N. Kalton, The Maharam problem, in: Séminaire d'Initiation à l'Analyse, Exp. No. 18, 13 pp., Publ. Math. Univ. Pierre et Marie Curie 94, Univ. Paris VI, Paris, 1989.

[10] J. Kelley, Measures on Boolean algebras, Pacific J. Math. 9 (1959), 1165-1177.

[11] D. Maharam, An algebraic characterization of measure algebras, Ann. of Math. (2) 48 (1947), 154-167.

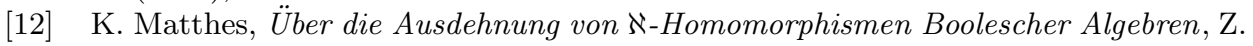
Math. Logik Grundlagen Math. 6 (1960), 97-105.

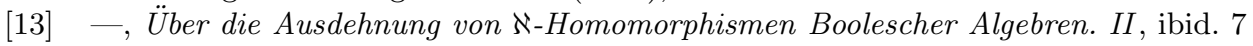
(1961), 16-19.

[14] J. von Neumann, Problem 163, July 4, 1937, in: The Scottish Book, R. D. Mauldin (ed.), Birkhäuser, Boston, MA, 1981.

[15] J. C. Oxtoby, Math. Reviews MR0161952 (28\#5156), January 1965.

[16] S. Quickert, CH and the Sacks property, Fund. Math. 171 (2002), 93-100.

[17] R. Sikorski, Boolean Algebras, Springer, 1964.

[18] S. Solecki, Analytic ideals and their applications, Ann. Pure Appl. Logic 99 (1999), $51-72$.

[19] A. Tarski, Ideale in vollständigen Mengenkörpern. I, Fund. Math. 32 (1939), 45-63.

[20] —, Ideale in vollständigen Mengenkörpern. II, ibid. 33 (1945), 51-65.

[21] S. Todorcevic, A dichotomy for P-ideals of countable sets, ibid. 166 (2000), 251-267.

[22] B. Velickovic, CCC forcing and splitting reals, CRM preprint no 569, 02/2004.

[23] D. A. Vladimirov, Boolean Algebras in Analysis, Math. Appl. 540, Kluwer, Dordrecht, 2002.

Université Paris VII, C.N.R.S.

UMR 7056

2 Place Jussieu

75251 Paris, France

E-mail: stevo@math.jussieu.fr

Department of Mathematics

University of Toronto

Toronto, Canada, M5S 3G3

E-mail: stevo@math.toronto.edu
Centre de Recerca Matèmatica Apartat 50

E-08193 Bellaterra, Spain

E-mail: stodorcevic@crm.es

Received 13 July 2004 